\title{
IVUS-Guided Zero-Contrast PCI in CKD Patients: Safety and Short-Term Outcome in Patients with Complex Demographics and/or Lesion Characteristics
}

\author{
Prathap Kumar (D), Blessvin Jino $(\mathbb{D}$, Ali Shafeeq, Stalin Roy, Manu Rajendran, \\ and Sandheep George Villoth $\mathbb{D}$
}

Department of Cardiology, Meditrina Hospital, Kollam, Kerala, India

Correspondence should be addressed to Prathap Kumar; prathapndr@gmail.com

Received 29 December 2020; Revised 26 April 2021; Accepted 3 May 2021; Published 8 May 2021

Academic Editor: Shenghua Zhou

Copyright (c) 2021 Prathap Kumar et al. This is an open access article distributed under the Creative Commons Attribution License, which permits unrestricted use, distribution, and reproduction in any medium, provided the original work is properly cited.

\begin{abstract}
Background. Percutaneous coronary intervention (PCI) in patients with significant renal dysfunction is challenging because of the lesion characteristics and the risk of contrast-induced acute kidney injury (CI-AKI). With the advent of intravascular ultrasound(IVUS-) guided zero-contrast PCI, outcomes have improved considerably. Objective. To assess the safety and short-term outcomes of IVUS-guided zero-contrast PCI in chronic kidney disease (CKD) patients with complex demographics or lesion morphology. Methods. Patients who underwent IVUS-guided zero-contrast PCI at a tertiary center, from November 2019 to May 2020, were included in this prospective analysis. Clinical characteristics, procedural data, and follow-up data were collected and analyzed. Results. A total of 15 patients ( 27 vessels), all men (mean age, $70.0 \pm 11.0$ years), underwent zero-contrast PCI. The mean estimated glomerular filtration rate (eGFR) and serum creatinine were $30.8 \pm 7.3 \mathrm{~mL} / \mathrm{min} / 1.73 \mathrm{~m}^{2}$ and $2.6 \pm 1.3 \mathrm{mg} / \mathrm{dL}$, respectively. The mean BMC2 risk for dialysis was $2.1 \pm 1.1 \%$, mean SYNTAX score was $20.3 \pm 10.3$, and mean left ventricular ejection fraction (LVEF) was $42.4 \pm 11.6 \%$. Four patients $(26.6 \%)$ underwent left main coronary artery (LMCA) PCI including one LMCA bifurcation. One patient underwent chronic total occlusion PCI. Technical and procedural success were 100\% without any periprocedural complications. No major adverse cardiovascular events (MACE) were reported, and no patient required dialysis within three months of follow-up. Conclusion. Zero-contrast PCI guided by IVUS is safe in coronary artery disease (CAD) patients with moderate-to-severe CKD. High procedural success without complications can be achieved even in cases with complex clinical characteristics and lesion morphology.
\end{abstract}

\section{Introduction}

Percutaneous coronary intervention (PCI) in older patients, those with underlying chronic kidney disease (CKD) and diabetes mellitus, has high risk of contrast-induced acute kidney injury (CI-AKI) $[1,2]$. Also, estimated glomerular filtration rate (eGFR) less than $60 \mathrm{~mL} / \mathrm{min} / \mathrm{m}^{2}$ is considered a strong risk factor for $\mathrm{CI}-\mathrm{AKI}$ during intra-arterial contrast administration. According to the Mayo Clinic registry, 3.3\% of patients who underwent PCI, developed AKI [3]. The incidence during primary PCI for acute myocardial infarction (MI) has been reported to be 19\% [4]. In a pooled analysis of the HORIZONS-AMI and ACUITY trials, CIAKI resulted in an increased risk of short- and long-term ischemic and vascular events in ACS patients undergoing PCI [5]. Further, the mortality rates at one month and one year were significantly higher in patients with CI-AKI compared to patients without CI-AKI. Data from the 13,349,612 patients from the American College of Cardiology National Cardiovascular Data Registry (NCDR), CathPCI Registry revealed that every incremental $75 \mathrm{~mL}$ of contrast use increased the risk of AKI by $42 \%$ [6]. In a modeling study, contrast volume exceeding creatinine clearance by 2.99 times was associated with a significant risk 
of AKI, while reduction of contrast volume by $30 \%$ prevented AKI incidence by $13 \%$ [7].

Several approaches-including prophylactic hydration, administration of high-dose statins, $N$-acetylcysteine, and usage of iso-osmolar agents-have been attempted to prevent CI-AKI [8]. However, none of these measures has been consistently beneficial, except hydration with normal saline. Though iso-osmolar contrast agents are used commonly, they are not completely safe and can be nephrotoxic as well [9]. Moreover, heavily calcified lesions in CKD patients pose several challenges during lesion preparation and stent delivery-necessitating higher contrast during the procedure. These patients are often denied PCI due to increased risk of CI-AKI. The advent of image-guided zero- or ultralow-contrast PCI is a boon for such patients.

Over the past few years, several authors have reported good procedural success in ultra-low-volume or zero-contrast PCI $[10,11]$. However, there is a paucity of data on zero-contrast PCI for complex lesion subsets such as left main disease, chronic total occlusions (CTO), and heavily calcified lesions. Hence, this study was conducted to determine the feasibility, safety, and clinical utility of IVUSguided zero-contrast PCI in CAD patients with underlying moderate-to-severe $\mathrm{CKD}$ and complex clinical or lesion characteristics.

\section{Methods}

2.1. Study Design and Population. This was a prospective single-center observational study. Clinical and procedural data were obtained from all consecutive patients who underwent zero-contrast PCI at our tertiary care center between November 2019 and May 2020. Percutaneous coronary intervention was planned in patients with significant stenosis (angiographic diameter stenosis $\geq 70 \%$ in nonLMCA and $\geq 50 \%$ in LMCA, IVUS measured minimal luminal area of $<6 \mathrm{~mm}^{2}$ in LMCA lesions, or flow fraction reserve $[F F R] \leq 0.8)$ and indication for revascularization. Patients underwent "zero-contrast PCI" if they had met any of the following criteria: (1) eGFR $<30 \mathrm{~mL} / \mathrm{min} / 1.73 \mathrm{~m}^{2}$; (2) $\mathrm{eGFR}<45 \mathrm{~mL} / \mathrm{min} / 1.73 \mathrm{~m}^{2}$ (Stage $3 \mathrm{~b}, 4$, and $5 \mathrm{CKD}$ ) among patients aged $>75$ years or with left ventricular ejection fraction $(\mathrm{LVEF})<35 \%$.

2.2. Procedures. A detailed history was collected along with baseline clinical characteristics and laboratory investigations. Baseline echocardiography and electrocardiographic changes were recorded before the procedure to facilitate the detection of changes during the procedure. Standard techniques and catheters were used during the PCI procedure. All procedures were carried out by a single operator with an experience of 200 LMCA PCI and 150 chronic total occlusion (CTO) PCI per year. Procedures were performed via femoral access and 7F guide catheters in all cases, except for one, where a $6 \mathrm{~F}$ catheter and radial access was used. Stenting strategy (particularly in bifurcation lesions), lesion preparation, the number of stents, and postdilatation were left to the operator's discretion. In general, rotational atherectomy was used when IVUS detected calcium arc $>180^{\circ}$ and calcium length $\geq 5 \mathrm{~mm}$. Postdilatation was performed mostly using noncompliant (NC) balloons. Informed consent was obtained from all patients before the procedure. Blood transfusion was planned if postprocedure hemoglobin had reduced to $8 \mathrm{gm} \%$. Boston scientific iLAB ultrasound imaging system with OptiCross 6 coronary imaging catheter (40 MHz) was used for IVUS runs. The study was approved by the institutional review board.

\subsection{Zero-Contrast Percutaneous Coronary Intervention} Protocol. Coronary angiogram (CAG) was performed using ultra-low-volume contrast (total contrast volume in $\mathrm{ml}$ was less than the eGFR in $\mathrm{mL} / \mathrm{min} / 1.73 \mathrm{~m}^{2}$ ). After angiography, guide catheter engagement was confirmed by passing the guidewire and identifying the wire course along the vessel in comparison with angiogram alongside the same fluoroscopic projection. Additional wires (hydrophobic or hydrophilic) were placed in the side branches to silhouette the main vessel and major side branches. With the guidance of IVUS across the main vessel and side branches (in left main cases), the lesion length, proximal and distal reference vessel diameters, calcium arc and length and landing zones were identified. Fluoroscopically, proximal and distal landing zones were identified by the length from the nearest side branch. "Cine store" was done during IVUS run to identify the landing zones. In the case of aorto-ostial lesions, "floating wire technique" was used. After the initial IVUS run, lesion preparation was done using a semicompliant balloon, scoring/cutting balloon, or rotational atherectomy, according to the lesion morphology. Repeat IVUS was done to assess the adequacy of lesion preparation and extent of dissection (if any) and confirm the measurements. Fluoroscopic projection was not changed during stent deployment. After stenting, IVUS run was done to detect significant edge dissection, stent underexpansion, malapposition, minimal stent areas (MSA), and ostial coverage. Postdilatation was done if needed. A serial echocardiogram was done to rule out pericardial effusion.

2.4. Definitions and Endpoints. Technical success was defined as minimal stent area (MSA) $>80 \%$ of average reference lumen with no significant edge dissections and plaque burden of $<50 \%$ at landing zones. Procedural success was defined as technical success without in-hospital mortality. Contrast-induced AKI was defined as an increase in serum creatinine by $\geq 0.3 \mathrm{mg} / \mathrm{dL}$ within $48 \mathrm{~h}$ after contrast medium exposure or a $\geq 50 \%$ increase within seven days (Kidney Disease: Improving Global Outcomes (KIDGO) working group definition). Blood transfusion was planned if postprocedure hemoglobin had reduced to $<8 \mathrm{gm} \%$. Stent thrombosis was defined as symptoms of acute coronary syndrome along with angiographic evidence of thrombus inside the stent.

All patients were followed up for three months. Check coronary angiogram with minimal dye was advised if the patient developed new angina, new significant electrocardiogram (ECG) changes, or hemodynamic instability. 
2.5. Statistical Analysis. No formal size calculation was done since this is an observational study. Categorical variables are presented as numbers and percentages and continuous data as mean and standard deviation.

\section{Results}

3.1. Baseline Patient Characteristics. Zero-contrast PCI was carried out in a total of 27 vessels in 15 patients during the study period. Table 1 shows the baseline clinical characteristics of the patients. The mean age of the patients was $70.0 \pm 10.0$ years, and all the patients were men. The majority of the patients (66.6\%) were aged $\geq 70$ years. Initial hospital presentation was SIHD in seven patients, non-ST-elevation myocardial infarction (NSTEMI) in four patients, and unstable angina and ST-elevation myocardial infarction (STEMI) in two patients each. In the STEMI patients, non-culprit lesions were addressed with zero-contrast PCI in the same admission before discharge (3-5 days after primary PCI). Thirteen (86.7\%) patients had a history of hypertension and diabetes mellitus while ten $(66.7 \%)$ patients had dyslipidemia. One patient was on maintenance hemodialysis. Ten patients presented with CKD stage $3 \mathrm{~b}$, four patients presented with CKD stage 4 , and one patient had end-stage renal disease (ESRD). The mean baseline serum creatinine and eGFR were $2.6 \pm 1.3 \mathrm{mg} / \mathrm{dL}$ and $30.8 \pm 7.3 \mathrm{~mL} / \mathrm{min} / 1.73 \mathrm{~m}^{2}$, respectively. The mean BMC2 dialysis risk was $2.1 \pm 1.1 \%$. Out of 15 patients, 3 patients underwent coronary angiogram at other centers. In those cases, zero-contrast PCI was done after one week. Two patients presented as STEMI and they underwent non-culprit vessel revascularization as zero-contrast PCI after 3-5 days. All the remaining ten cases underwent ad hoc zerocontrast PCI at our center.

3.2. Lesion and Procedural Characteristics. Single-vessel, twovessel, and three-vessel disease were noted in two, four, and nine patients, respectively. The mean number of vessels involved was $1.7 \pm 0.7$ (Table 2). Chronic total occlusion was present in one patient. Significant LMCA disease was seen in four patients. The mean SYNTAX I and SYNTAX II scores were $20.3 \pm 10.3$ and $45.6 \pm 7.2$, respectively. Calcified lesions were detected in 19 vessels with calcium arc $>180^{\circ}$ seen in 6 vessels. The mean lesion length was $37.4 \pm 17.1 \mathrm{~mm}$. Stenting was done in 26 vessels and balloon angioplasty with drug coated balloon in one vessel. Most of the patients received a single stent, with both pre- and postdilatation (Table 2). Four patients had significant LMCA disease; out of those, three patients underwent LMCA-LAD stenting, and one patient underwent LMCA bifurcation stenting. Rotational atherectomy ( $1.5 \mathrm{~mm}$ burr) was required in two patients. The mean value of minimal stent area of LMCA was $12.4 \pm 1.8 \mathrm{~mm}^{2}$ (Table 3). All procedures were completed with $0 \mathrm{~mL}$ of contrast, a mean fluoro time of $30.8 \pm 8.3$ minutes, and a mean radiation dosage of $2933.5 \pm 1098.9 \mathrm{mGy}$.

3.3. Clinical Outcomes. Technical and procedural success were achieved in all patients $(100 \%)$. There was no death, MI, or stent thrombosis during hospital stay. There were no adverse events, including pericardial effusion, edge
TABLE 1: Baseline clinical characteristics.

\begin{tabular}{lc}
\hline Baseline characteristics & Overall group $(n=15)^{*}$ \\
\hline Age (mean \pm SD) (years) & $70.0 \pm 11.0$ \\
Male & $15(100 \%)$ \\
Hypertension & $13(86.7 \%)$ \\
Diabetes mellitus & $13(86.7 \%)$ \\
Dyslipidemia & $10(66.7 \%)$ \\
Previous PCI & $4(26.7 \%)$ \\
Previous CAD & $5(33.3 \%)$ \\
Initial hospital presentation & \\
SIHD & $7(46.6 \%)$ \\
$\quad$ Unstable angina & $2(13.3 \%)$ \\
NSTEMI & $4(26.6 \%)$ \\
STEMI (non-culprit lesion) & $2(13.3 \%)$ \\
$\quad$ LVEF (mean \pm SD) & $42.4 \pm 11.6$ \\
\hline LVEF (\%) & \\
$>50$ & $4(26.6 \%)$ \\
$40-49$ & $6(40 \%)$ \\
$\quad<40$ & $5(33.3 \%)$ \\
Serum creatinine $(\mathrm{mg} / \mathrm{dL})(\mathrm{mean} \pm \mathrm{SD})$ & $2.6 \pm 1.3$ \\
eGFR (mL/min/1.73 m $\left.{ }^{2}\right)($ mean \pm SD) & $30.8 \pm 7.3$ \\
BMC2 CIN risk $(\%)($ mean \pm SD) & $15.3 \pm 8.3$ \\
BMC2 dialysis risk $(\%)($ mean \pm SD) & $2.1 \pm 1.1$ \\
\hline
\end{tabular}

${ }^{*}$ All data are presented as $n$ (\%) unless otherwise specified. BMC:Blue Cross Blue Shield of Michigan Cardiovascular Consortium; CIN : contrastinduced nephropathy; eGFR: estimated glomerular filtration rate; LVEF: left ventricular ejection fraction; NSTEMI: non-ST-elevation myocardial infarction; PCI : percutaneous coronary intervention; SD : standard deviation; SIHD : stable ischemic heart disease; STEMI : ST-elevation myocardial infarction.

TABle 2: Angiographic and IVUS characteristics.

\begin{tabular}{lc}
\hline Angiographic and IVUS data & Overall group \\
\hline Number of PCI vessels $(n)$ & 27 \\
Number of vessels involved per patient & $1.7 \pm 0.7$ \\
(mean \pm SD) & $20.6 \pm 10.0$ \\
SYNTAX I score $($ mean \pm SD) $(n=15)$ & \\
SYNTAX I score & $8(53.3 \%)$ \\
$\quad<23$ & $6(40 \%)$ \\
$23-32$ & $1(6.7 \%)$ \\
$>32$ & $45.4 \pm 6.9$ \\
SYNTAX II score $($ mean \pm SD) $(n=15)$ & \\
Calcium arc & $6(22.2 \%)$ \\
$\quad \geq 180^{\circ}$ & $13(48.1 \%)$ \\
$<180^{\circ}$ & $8(29.6 \%)$ \\
No calcium
\end{tabular}

${ }^{*}$ All data are presented as $n$ (\%) unless otherwise specified; $n$ varied in a few cases and has been specified accordingly. IVUS : intravascular ultrasound; LMCA: left main coronary artery; MLA:minimal luminal area; POC: polygon of confluence; PCI : percutaneous coronary intervention; SYNTAX: Synergy between Percutaneous Coronary Intervention with Taxus and Cardiac Surgery; SD : standard deviation.

dissection, vascular complications, urgent coronary artery bypass surgery (CABG) requirement, or major bleeding events requiring blood transfusion, during hospital stay. Further, at a median follow-up period of 90 days, all patients were asymptomatic and stable without any MACE events; there was no significant increase in 
TABle 3: Procedural characteristics.

\begin{tabular}{|c|c|}
\hline Procedural data & Overall group* \\
\hline Number of vessels treated with zero-contrast PCI $(n)$ & 27 \\
\hline \multicolumn{2}{|l|}{ Approach $(n=15)$} \\
\hline Femoral & $14(93.3 \%)$ \\
\hline Radial & $1(6.7 \%)$ \\
\hline \multicolumn{2}{|l|}{ Guide Catheter $(n=15)$} \\
\hline $7 \mathrm{~F}$ & $14(93.3 \%)$ \\
\hline $6 \mathrm{~F}$ & $1(6.7 \%)$ \\
\hline Number of wires per vessel (mean $\pm S D)$ & $2.5 \pm 0.9$ \\
\hline Number of IVUS runs per vessel (mean \pm SD) & $3.3 \pm 0.9$ \\
\hline Microcatheters used & $2(7.4 \%)$ \\
\hline Number of stents per vessel (mean \pm SD) & $1.4 \pm 0.6$ \\
\hline LMCA PCI $(n)$ & 4 \\
\hline LMCA bifurcation PCI & $1(3.7 \%)$ \\
\hline Rotational atherectomy & $2(7.4 \%)$ \\
\hline Chronic total occlusion PCI & $1(3.7 \%)$ \\
\hline ISR PCI & $1(3.7 \%)$ \\
\hline Minimal stent area non-LMCA $\left(\mathrm{mm}^{2}\right)($ mean \pm SD $)(n=27)$ & $6.5 \pm 2.6$ \\
\hline Minimal stent area LMCA $\left(\mathrm{mm}^{2}\right)($ mean \pm SD $)(n=27)$ & $12.4 \pm 1.8$ \\
\hline Fluoroscopy time $(\min )($ mean $\pm \mathrm{SD})$ & $30.8 \pm 8.3$ \\
\hline Radiation dose $(\mathrm{mGy})($ mean $\pm \mathrm{SD})$ & $2933.5 \pm 1098.9$ \\
\hline Technical success & $100 \%$ \\
\hline Procedural success & $100 \%$ \\
\hline Peak/48-hour creatinine $($ mean $\pm \mathrm{SD})(\mathrm{mg} / \mathrm{dL})$ & $2.4 \pm 1.2$ \\
\hline
\end{tabular}

creatinine levels or eGFR, and none of the patients required dialysis.

\section{Discussion}

Individuals with moderate-to-severe renal dysfunction (creatinine clearance $<60 \mathrm{~mL} / \mathrm{min}$ ) are especially vulnerable to the adverse CI-AKI related events [12]. This prospective observational single-center study evaluated the feasibility and safety of IVUS-guided zero-contrast PCI in ACS and SIHD patients with moderate-to-severe CKD. The study confirms that IVUS-guided zero-contrast PCI is a safe and effective strategy for coronary intervention even in patients with many high-risk features.

Though the major benefit of zero-contrast PCI is prevention of CI-AKI, it has other benefits too. Patients with severe LV systolic dysfunction frequently develop pulmonary edema on contrast administration since contrast media-related hyperosmolality and functional hypocalcemia depress the myocardial contractility. [13] Also, elderly patients, particularly $>75$ years, have decreased functional renal reserve and they may not tolerate the minimal renal injury by contrast which "young people with borderline renal function" frequently tolerate. [14] Hence, our institutional criteria for zero-contrast PCI was not only eGFR $<30$, but also included elderly and patients with severe LV systolic dysfunction-mean age was 70 years (4 patients were $>80$ years of age); LV ejection fraction $<40 \%$ in $33.3 \%$ of patients (3 patients had $\operatorname{LVEF}^{\circ}<30 \%$ ). Hence, even though mean was eGFR $\sim 30.8$, many patients underwent zero-contrast PCI. As per the BMC2 CIN risk calculator, $<1 \%$ CIN risk is called low risk, $1-7 \%$ is called intermediate risk, and $>7 \%$ is called high risk. Our cohort had mean BMC2 CIN risk score of $15.3 \pm 8.3$ which substantiated the need for zero-contrast PCI. All patients had moderate-tosevere CKD (stages 3b, 4 , and 5) and dialysis risk was $2.1 \%$. Furthermore, the lesion morphologies were also complex with SYNTAX score $\geq 23$ in $53.3 \%$ of patients, heavy calcification ( $\geq 180$-degree calcium arc) in $22 \%$ of the vessels, CTO in one vessel, and LMCA involvement in four patients. Despite these high-risk features, $100 \%$ technical and procedural success rates were achieved. Similar to our study, a retrospective analysis demonstrated $100 \%$ procedural success in patients with advanced CKD $(n=24)$ who underwent zero-contrast PCI [10]. In another recent retrospective analysis, a total of 29 patients-including 20 patients with advanced CKD and four patients on hemodialysis-successfully underwent zero-contrast PCI without worsening of renal function [11]. In the current study, four patients with advanced CKD and one patient on dialysis underwent zero-contrast PCI successfully. Further, the eGFR and ejection fraction improved in the patient on dialysis postintervention, and dialysis was no longer required.

All procedures except for one in the current study were performed using transfemoral approach and $7 \mathrm{~F}$ catheter. Transfemoral approach is preferred because we wanted to preserve radial artery for future dialysis. Many CKD patients have complex lesion morphologies, which may need rotablation, guide-extension catheters, etc. Moreover, we needed 
multiple marker wires to precisely position the stent in zerocontrast PCI; hence, 7F guide-extension catheter was a better option in most cases.

Intracoronary imaging, particularly IVUS, is an important component of zero-contrast PCI. Expertise in IVUS image interpretation and strict vigilance in anticipating, identifying, and successfully managing complications is of paramount importance in zero-contrast PCI. For all the procedures in the current study, previous coronary angiograms were used as a roadmap to pass the guidewires distally. The stents were selected according to IVUS measurements and deployed based on landmarks. In general, during the IVUS pull-back, "dry cine" can be taken to locate the proximal and distal landing zones (Figure 1). The other technique to identify the "landing zones" fluoroscopically is by parking guidewires in major branches and measuring the length from such branching points to the stent landing zone ("metallic silhouette" technique) [15]. In our study, the "metallic silhouette" technique was used in most vessels to place the stent in "landing zones" selected by IVUS (Figure 2).

Precise positioning of the stent in the vessel ostium was one of the major challenges noted in the current study; it is especially difficult to check the exact stent position and perform aorto-ostial stenting without contrast. "Sepal wire technique" was used for stent positioning across the LMCA and right coronary artery (RCA) ostia (Figure 3). Ostial stenting was planned in eleven vessels ( $3 \mathrm{RCA}$; $6 \mathrm{LAD}$; and 2 LM-LAD) and in all cases except one, ostia were successfully covered with a stent using marker wires (Figure 2). Further, a case of LMCA bifurcation PCI was successfully carried out by "mini-crush" technique. While using two stents in a vessel, minimal overlap was done using "ClearStent" technology from Siemens.

Two patients underwent plaque modification using rotational atherectomy (burr size $1.5 \mathrm{~mm}$ in both cases) in the current study. No reflow/slow flow is not uncommon after rotational atherectomy due to distal microembolization. Optimal antiplatelet, anticoagulation medication, and continuous use of flush cocktail can prevent this complication [16]. In the absence of contrast injection, meticulous assessment of patient symptoms, ECG changes (e.g., bradycardia or ST-elevation), hemodynamic instability, and new regional wall motion abnormality is necessary. Another major complication expected during rotational atherectomy is coronary perforation [16]. In cases where complications are suspected by new symptoms, ECG changes, or hemodynamic instability, an immediate check angiogram should be done with minimal contrast. In our study, rotational atherectomy was performed in two vessels without any such complication.
There was no incidence of death during the study period. Postprocedurally, none of the patients had an increase in creatinine levels. Furthermore, none of the patients developed pericardial effusion, stent thrombosis or required repeat revascularization during hospital stay or at three months of follow-up. In line with the current study, no MACE or renal replacement therapy, at a median follow-up of 79 days [10] and no events of ACS, stent thrombosis, and repeat revascularization at a median follow-up of 3.2 months [11] were observed in retrospective studies.

Another retrospective analysis compared eight ultralow-contrast (ULC) PCI with 103 conventional PCI in patients with high or very high CI-AKI risk score and advanced CKD not on dialysis. The study had no angiographic or procedural exclusion criteria and included CTO-PCI, rotational atherectomy, and left main bifurcation. Technical success was seen in $88 \%$ of cases with $0 \%$ CI-AKI with a fluoroscopy time of 39 minutes [17]. Similarly, the current study has included a single case of CTO, four cases of left main bifurcation, and two cases of rotational atherectomy. However, the mean fluoroscopy time was almost 9 minutes less than that seen in ULC-PCI study. Most importantly, we did not observe any significant coronary dissection, pericardial effusion, vascular complications, and no reflow without any contrast. While ULC-PCI study observed that small dose of contrast at the end of procedure will aid the operator with wealth of benefits in the treated region, a recent observational study in complicated ACS syndrome has observed the incidence of CI-AKI in few patients even with $3-5 \mathrm{ml}$ of contrast. [18].

The inclusion of patients with complex clinical characteristics or lesion morphologies is the unique feature of our study. The outcomes are proof of concept that even highly complex cases such as left main bifurcation disease and chronic total occlusion can be managed successfully with the help of IVUS-guided zero-contrast PCI. Rotational atherectomy and aorto-ostial stenting, which usually require contrast, were successfully performed without contrast. When done by experts, zero-contrast PCI can fulfil the "unmet" need of complex PCI in patients with significant renal impairment who are otherwise ineligible for routine PCI for fear of worsening renal function.

This study had few limitations. The study population was small, and hence feasibility and safety should be ascertained in a larger population. Further studies involving larger sample sizes are warranted to ascertain the long-term outcomes of IVUS-guided zero-contrast PCI. Secondly, our study was an observational study. Randomized control trials are needed to know the outcome this method comparing conventional PCI using iodinated contrast. Thirdly, long- 


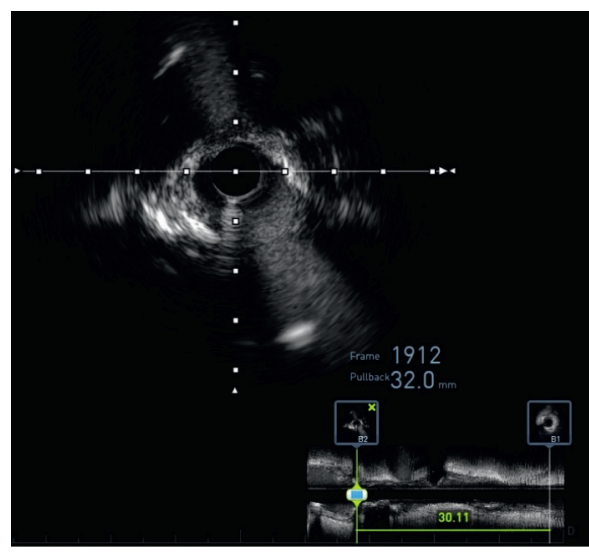

(a)

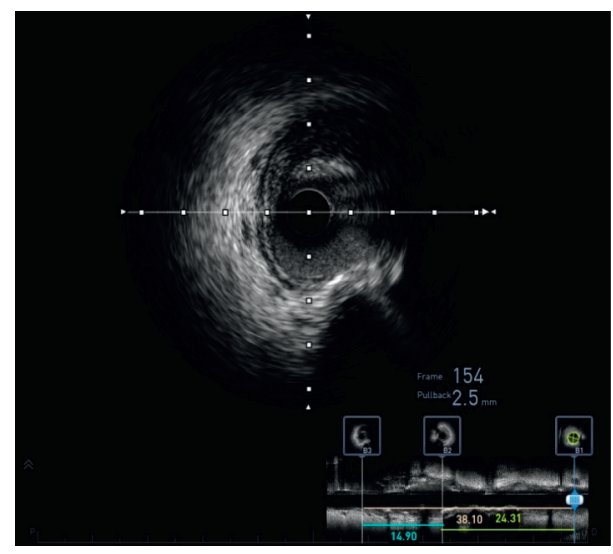

(b)

FIGURE 1: (a) IVUS image showing lesion length measurement from ostium of LAD to distal landing zone. In the IVUS picture above, we can see the wire in the LCX which is relatively free of disease. (b) Length measurements when two stents are planned, particularly when there is gross size discrepancy between proximal and distal landing zones.

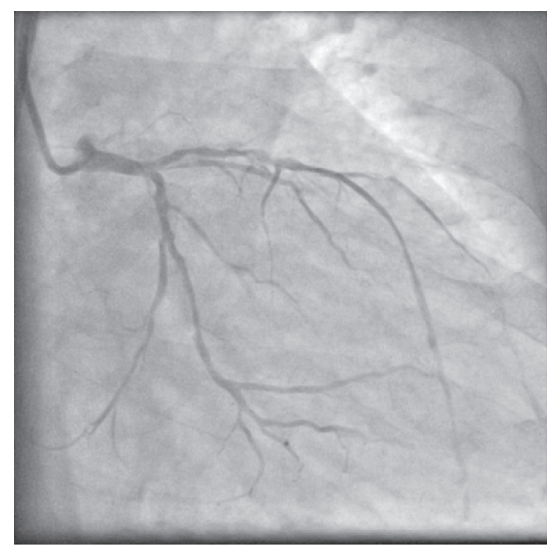

(a)

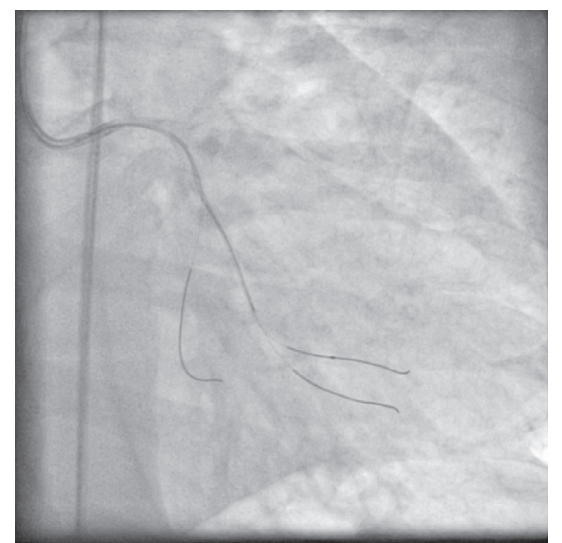

(b)

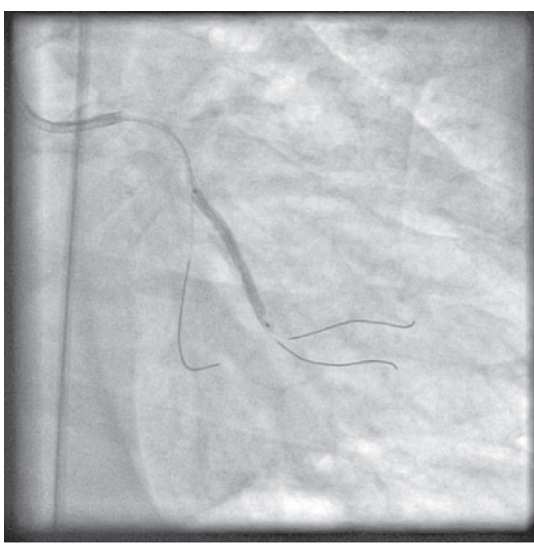

(c)

FIGURE 2: (a) RAO caudal view showing significant disease in LCX, OM, and LAD. (b) Metallic silhouette created by wiring the two branches of OM and distal LCX. (c) Stent positioned using the marker wires.

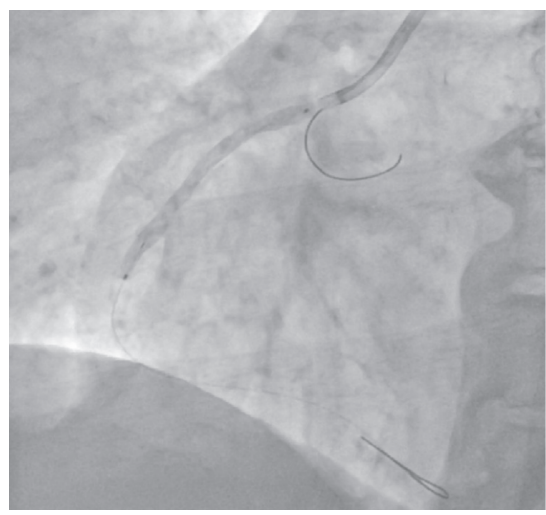

(a)

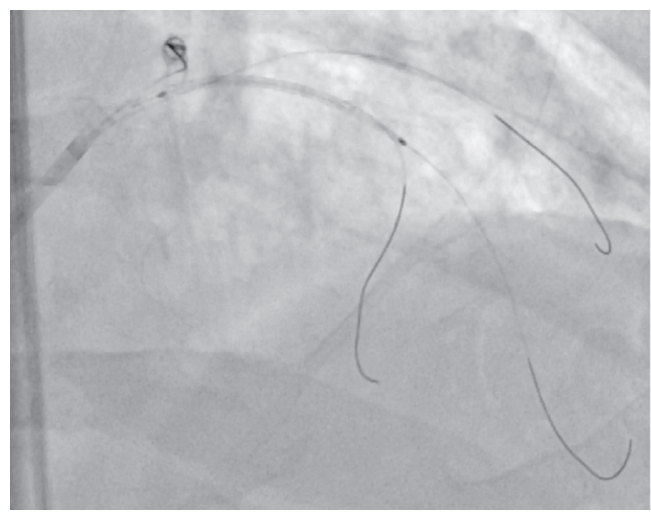

(b)

FIgURE 3: (a) LAO cranial view showing the wire over the aorta to mark the RCA ostium and ostial positioning of stent. Note. The loop is created in the wire at RPDA. (b) Stent positioned in LAD according to the marker wires at septal, diagonal, and LCX. 
term outcomes of zero-contrast PCI are not known. Since the procedure was done without any contrast, final angiographic result is not known. Hence, long-term outcome studies are needed before practicing this technique in a large scale of population.

\section{Conclusion}

IVUS-guided zero-contrast PCI was found to be feasible and safe in CAD patients with moderate-to-severe CKD when done by experts. This technique can be used safely in patients who are at high risk for CI-AKI, in centers where there is expertise for the performance of complex PCI with intravascular imaging guidance.

\section{Data Availability}

Data are available from the corresponding author only upon request.

\section{Conflicts of Interest}

The authors declare that they have no conflicts of interest regarding the publication of this paper.

\section{Acknowledgments}

The authors would like to thank BioQuest Solutions for data analysis and editorial services.

\section{References}

[1] M. Milojevic, S. J. Head, M. J. Mack et al., "The impact of chronic kidney disease on outcomes following percutaneous coronary intervention versus coronary artery bypass grafting in patients with complex coronary artery disease: five-year follow-up of the SYNTAX trial," EuroIntervention, vol. 14, no. 1, pp. 102-111, 2018.

[2] T. Gupta, N. Paul, D. Kolte et al., "Association of chronic renal insufficiency with in-hospital outcomes after percutaneous coronary intervention," J Am Heart Assoc, vol. 4, no. 6, Article ID e002069, 2015.

[3] C. S. Rihal, M. D. Rihal, D. E. GrillTextor et al., "Incidence and prognostic importance of acute renal failure after percutaneous coronary intervention," Circulation, vol. 105, no. 19, pp. 2259-2264, 2002.

[4] G. Marenzi, G. Lauri, E. Assanelli et al., "Contrast-induced nephropathy in patients undergoing primary angioplasty for acute myocardial infarction," Journal of the American College of Cardiology, vol. 44, no. 9, pp. 1780-1785, 2004.

[5] D. Giacoppo, M. V. Madhavan, U. Baber et al., "Impact of contrast-induced acute kidney injury after percutaneous coronary intervention on short- and long-term outcomes: pooled analysis from the HORIZONS-AMI and ACUITY trials," CircCardiovascInterv, vol. 8, no. 8, Article ID e002475, 2015.

[6] A. P. Amin, R. G. Bach, M. L. Caruso, K. F. Kennedy, and J. A. Spertus, "Association of variation in contrast volume with acute kidney injury in patients undergoing percutaneous coronary intervention," JAMA Cardiology, vol. 2, no. 9, pp. 1007-1012, 2017.
[7] H. S. Gurm, M. Seth, R. Mehran et al., "Impact of contrast dose reduction on incidence of acute kidney injury (AKI) among patients undergoing PCI: a modeling study," The Journal of Invasive Cardiology, vol. 28, no. 4, pp. 142-146, 2016.

[8] D. Patschan, I. Buschmann, and O. Ritter, "Contrast-induced nephropathy: update on the use of crystalloids and pharmacological measures," International Journal of Nephrology, vol. 2018, Article ID 5727309, 8 pages, 2018.

[9] B. Mruk, "Renal safety of iodinated contrast media depending on their osmolarity - current outlooks," Polish Journal of Radiology, vol. 81, pp. 157-165, 2016.

[10] Z. A. Ali, K. Karimi Galougahi, T. Nazif et al., "Imaging- and physiology-guided percutaneous coronary intervention without contrast administration in advanced renal failure: a feasibility, safety, and outcome study," European Heart Journal, vol. 37, no. 40, pp. 3090-3095, 2016.

[11] J. Sacha, M. Gierlotka, P. Lipski, P. Feusette, and D. Dudek, "Zero-contrast percutaneous coronary interventions to preserve kidney function in patients with severe renal impairment and hemodialysis subjects," Advances in Interventional Cardiology, vol. 15, no. 2, pp. 137-142, 2019.

[12] M. Maioli, A. Toso, M. Leoncini, M. Gallopin, N. Musilli, and F. Bellandi, "Persistent renal damage after contrast-induced acute kidney injury," Circulation, vol. 125, no. 25, pp. 3099-3107, 2012.

[13] ACR Committee on Drugs and Contrast Media, ACR Manual on Contrast Media 2020, ACR Committee on Drugs and Contrast Media, 978-1-55903-012-0, 2020.

[14] C. G. Musso and D. G. Oreopoulos, "Aging and physiological changes of the kidneys including changes in glomerular filtration rate," Nephron Physiology, vol. 119, no. s1, pp. p1-p5, 2011.

[15] J. Sacha, "Marking wire technique for zero-contrast percutaneous coronary interventions," Advances in Interventional Cardiology, vol. 14, no. 2, pp. 204-205, 2018.

[16] T. Gupta, M. Weinreich, M. Greenberg, A. Colombo, and A. Latib, "Rotational atherectomy: a contemporary appraisal," Interventional Cardiology Review, vol. 14, no. 3, pp. 182-189, 2019.

[17] L. Azzalini, A. Laricchia, D. Regazzoli et al., "Ultra-low contrast percutaneous coronary intervention to minimize the risk for contrast-induced acute kidney injury in patients with severe chronic kidney disease," The Journal of Invasive Cardiology, vol. 31, no. 6, pp. 176-182, 2019.

[18] S. Higuchi, Y. Kabeya, Y. Nishina, Y. Miura, and H. Yoshino, "Feasibility and safety of noncontrast percutaneous coronary intervention in patients with complicated acute coronary syndrome," Catheterization and Cardiovascular Interventions, vol. 96, no. 7, pp. E666-E673, 2020. 